\title{
La relación entre los enfoques didácticos y la condición social de los estudiantes. Un problema clave en el debate educativo actual
}

\author{
Relationship between teaching approaches and social \\ condition of students: A key problem in the current \\ educational debate
}

\author{
Feldman, Daniel; Gori, Agustín; Diuk, Beatriz
}

Daniel Feldman Sobre el autor

danielf51@yahoo.com.ar

Instituto de Investigaciones en Ciencias de la

Educación (IICE), Argentina

Agustín Gori Sobre el autor agustingori7@gmail.com Consejo Nacional de Investigaciones Científicas y

Técnicas (CONICET - IICE), Argentina

Beatriz Diuk Sobre la autora beadiuk@gmail.com Universidad Nacional de San Martín (LICH UNSAM), Argentina

Itinerarios educativos

Universidad Nacional del Litoral, Argentina

ISSN: $1850-3853$

ISSN-e: $2362-5554$

Periodicidad: Anual

vol. 1, núm. 14, 2021

revistadelindi@fhuc.unl.edu.ar

Recepción: 14 Diciembre 2020

Aprobación: 06 Agosto 2021

URL: https://doi.org/10.14409/ie.2021.14.e0008

Esta obra está bajo una Licencia Creative Commons AtribuciónNoComercial-CompartirIgual 4.0 Internacional.
Resumen: Desde hace varios años en América Latina aumentaron las preocupaciones en torno a la desigualdad y a la exclusión educativa que ella provoca. Los efectos positivos de distintas políticas destinadas a garantizar la inclusión educativa no ocultan el hecho de que todavía las dificultades que enfrentan los niños y las niñas provenientes de sectores populares continúan siendo muy importantes. En este trabajo se proponen algunas reflexiones sobre este problema centradas, principalmente, en las propuestas didácticas para el nivel primario en Argentina. Basada en la clásica distinción entre pedagogías «visibles e invisibles» la exposición está apoya en tres afirmaciones: En primer lugar, es necesario poner en cuestión la idea de que el enfoque didáctico que se adopte es neutral con relación a la condición social de los estudiantes. En segundo término, es necesario prestar atención a un amplio cuerpo de evidencia internacional que apoya las ventajas de la instrucción directa frente a los enfoques didácticos «centrados en el estudiante $\gg$ o «exploratorios $\gg$. Por último, es necesario revisar el supuesto de que, dada la supuesta neutralidad de los enfoques didácticos, el acceso a los beneficios de una buena enseñanza depende de compensar posibles déficits, por ejemplo, de «capital cultural».

Palabras clave: didáctica, sector social, pedagogía visible e invisible, rendimiento escolar.

Abstract: In the last decades, Latin America has been increasingly concerned with inequality and the consequent educational segregation. Various policies were adopted to ensure educational inclusion and they had positive effects. However, the latter do not prevent children from low-income sectors to still face considerable difficulties. In this paper, some reflections on this problem are proposed, which are mainly focused on didactic proposals for elementary school in Argentina. Based on the classic distinction between 'visible and invisible' pedagogies, the exposition is supported by three statements. First, it is necessary to question the idea that the adopted didactic approach is neutral in relation to the social condition of students. Second, it is worth considering a large body of international evidence that supports the advantages of direct instruction over 'student-centered' or 'exploratory' approaches to 
teaching. Finally, it is necessary to review the assumption that, given the supposed neutrality of didactic approaches, the access to the benefits of good quality teaching depends on compensating for possible deficits -for example, in 'cultural capital'-.

Keywords: instruction theory, social sector, visible and invisible pedagogies, school achievement.

\section{Introducción}

Desde hace varios años en nuestro país y en América Latina aumentaron las preocupaciones en torno a la desigualdad y a la exclusión educativa que ella provoca. Así es cómo se llevaron adelante distintas políticas destinadas a garantizar la inclusión educativa que se convirtió en un lema principal de las dos últimas décadas. Estas políticas operaron sobre las condiciones de partida, trataron de compensar factores que dificultaban el acceso a la actividad escolar, desarrollaron estrategias de mantenimiento del esfuerzo educativo, dispositivos para favorecer mejores trayectorias y distintas maneras de garantizar la terminalidad. Sus efectos positivos no ocultan el hecho de que la mayor inclusión en la actividad escolar no fue una garantía para obtener una mejora significativa en los niveles de aprendizaje de los niños y de las niñas provenientes de sectores populares. Estas persistentes dificultades pueden implicar un llamado de atención sobre las características de los enfoques didácticos utilizados, de los principios pedagógicos más generales con los que se trata de orientar la actividad escolar y sobre su papel en las oportunidades de los estudiantes.

En lo que sigue, queremos proponer algunas reflexiones sobre este problema centradas, principalmente, en las orientaciones con relación a las propuestas didácticas para el nivel primario en nuestro país, aunque entendemos que pueden tomarse en cuenta para explorar la situación de otros niveles y otros países latinoamericanos.

La exposición está basada en tres afirmaciones:

Primero, es necesario poner en cuestión la idea de que el enfoque didáctico que se adopte, las estrategias específicas que se utilicen y el marco pedagógico que las sustente son neutrales con relación a la condición social de los estudiantes (y a otras condiciones particulares frente al aprendizaje, pero ese no será el foco de este trabajo).

Segundo, es necesario prestar atención a un amplio cuerpo de evidencia internacional que apoya las ventajas de la instrucción directa frente a los enfoques didácticos «centrados en el estudiante»o «exploratorios».

Tercero, ligado con los dos puntos anteriores, es necesario poner en cuestión el supuesto de que, dada la supuesta neutralidad de los enfoques didácticos, para que todos accedan a los beneficios de una buena enseñanza es necesario compensar algunos posibles déficits, por ejemplo, de «capital cultural».

Propondremos algunos elementos conceptuales y referencias de investigaciones que nos permitan sostener la pertinencia de estas afirmaciones. Antes es necesario realizar una aclaración. Las tres afirmaciones planteadas colocan el problema en términos de enfoques didácticos, pero, como se verá de inmediato, el argumento se basa inicialmente en la descripción de dos pedagogías 
que son resultado de una modelización. En este trabajo utilizaremos tanto el término de «didáctica» como el de «pedagogía» como conceptos que, en distintos niveles de análisis, refieren sustancialmente a las características de la comunicación educativa en contexto. Puede decirse que «pedagogía» expresa principios generales y que «enfoque didáctico» alude a configuraciones que modelan un ordenamiento particular del conocimiento a enseñar, una pauta de actividad y un sistema específico de comunicación e interacciones en el aula. Un enfoque didáctico puede expresarse en distintas propuestas metodológicas, que, pese a sus diferencias, pueden ser encuadradas analíticamente dentro de las características básicas del enfoque y/o de los principios básicos de la pedagogía.

\section{¿Es neutral el enfoque didáctico con relación a la condición social de los estudiantes?}

La vinculación entre los principios que rigen la comunicación educativa y las condiciones de clase de los estudiantes fue planteada hace más de cuarenta años por Basil Bernstein, quien postuló la existencia de dos tipos de pedagogías que habilitaban o dificultaban de distinta manera la relación con la tarea escolar de los diversos sectores sociales. Bernstein planteó que, a partir de las décadas de 1960 y 1970, comenzaron a consolidarse en los niveles de educación inicial y primario formas de relación pedagógica que desafiaban a los patrones más tradicionales basados en reglas explícitas, una demarcación fuerte de los contenidos, un control alto sobre la secuencia y el ritmo de trabajo y claros criterios de desempeño y evaluación. En estas nuevas propuestas de enseñanza la tarea del docente consistía en disponer un ambiente que los estudiantes debían explorar, más que en presentar de manera clara el contenido. Esos ambientes se caracterizaban por una menor clasificación, estaban regulados por normas menos fuertes y regidos por un control más implícito que explícito por parte del profesor. Se ponía menor énfasis en la transmisión y adquisición de destrezas específicas. Las denominó «pedagogías invisibles», frente a los tradicionales enfoques de «pedagogías visibles» (Bernstein, 1988).

Según Bernstein, la emergencia de las pedagogías invisibles conjugaba con nuevos patrones de socialización y crianza propios de lo que llamaba la «nueva clase media», ligada con la actividad profesional, los servicios y, en general, la producción simbólica más que la producción material. Al mismo tiempo, creaba pautas de interacción, comunicación y actividad en el aula más ajenas a la experiencia de los estudiantes provenientes de los sectores populares que dominaban otros principios de comunicación, y de sus familias, que perdían capacidad de intervenir en el esfuerzo educativo de sus hijos.

Las afirmaciones de Bernstein agregaron un elemento crucial a la discusión sobre la enseñanza: posicionaron en términos sociales las diferentes capacidades para relacionarse con la demanda escolar. Ya no se trataría solo de diferencias individuales, vinculadas con características personales o cognitivas. Aunque esto ya era ampliamente reconocido por la sociología de la educación en ese momento, el planteo de Bernstein incorporó un elemento más: puso el foco en las relaciones diferenciales que establecen los estudiantes de distintos sectores sociales con el ambiente configurado por los principios que regulan la actividad pedagógica (el «código» como veremos más adelante) y situó estas diferencias en dos aspectos 
principales. El primero de ellos, dicho de un modo muy simplificado, es el hecho de que algunos estudiantes han sido socializados en los requerimientos que rigen esos ambientes educativos y otros no. El segundo consiste en que solo las familias de clase media pueden aportar la «práctica pedagógica doméstica» (Bernstein, 1993:93) requerida para transitar con éxito un modelo de pedagogía invisible. Estos dos factores implican una diferencia sustancial, ya que los principios de las pedagogías visibles, sus regla, textos y expectativas de logro (la respuesta acerca de qué debe ser hecho, con qué, a qué ritmo y para lograr qué tipo de cosas) son explícitos y, por lo tanto, disponibles para los participantes de la actividad educativa. De allí que Bernstein afirme que: «(...) las características superficiales de la pedagogía visible pueden ser entendidas por todos, dado que constituyen la forma pedagógica normal, se reproduzcan o no, en las familias sus principios y prácticas subyacentes» (Bernstein, 1993:93). En tanto que los principios de la pedagogía invisible, por ser tácitos y descansar en la regulación que el maestro u otros adultos realizan sobre la actividad y los procesos de las niñas y los niños, son dominados, también tácitamente, solo por quienes han sido socializados en ellos. Por eso, quienes provienen de la clase media pueden, en general, transitar de un modelo pedagógico a otro ${ }^{1}$ y sus compañeros provenientes de los sectores populares experimentan dificultades con los enfoques didácticos enmarcados en el modelo de pedagogía invisible. Es necesario tener en cuenta que, en principio, con cualquier modelo los estudiantes de las clases más desfavorecidas obtienen peores resultados y encuentran muchas más dificultades en sus trayectos escolares, ya que ello es un rasgo propio de una sociedad clasista y desigual. Sin embargo, Bernstein indica que «Es importante señalar que la pedagogía visible no es intrínsecamente un transmisor para la reproducción de resultados escolares diferenciales entre alumnos de distintas clases sociales. En efecto, es posible crear una pedagogía visible que reduzca la relación entre clase social y resultados educativos». Esto exige cambios importantes, aunque no imposibles. Más adelante retomaremos este punto. Sin embargo, agrega «(...) es probable que la pedagogía invisible cree un código pedagógico intrínsecamente más difícil, al menos en principio, para que los grupos sociales en situaciones más desventajosas (desde la perspectiva de la educación formal) lleguen a leer y controlar» (Bernstein, 1993:88).

Todo enfoque didáctico supone la creación de un ambiente específico que define de alguna manera el valor y orientación del contenido y de sus relaciones internas y externas, establece un sistema de comunicación y de control, una secuencia de tareas, un ritmo y una indicación acerca de la actividad de los alumnos y de quien enseña o facilita el aprendizaje, según sea la opción elegida. Es así, que todo enfoque didáctico puede ser leído en el marco de un modelo más general como es el de «pedagogías visibles e invisibles». La perspectiva delineada hasta aquí sugiere que, de ninguna manera, puede considerarse a los enfoques didácticos y a las estrategias de enseñanza como neutrales con relación a la condición social de los estudiantes y que sus efectos pueden ser distintos a los esperados, algo que va más allá de las intenciones de quienes los promuevan. Esto requiere, a nuestro juicio, una evaluación profunda de los formatos de enseñanza con los que se trata de resolver o, al menos, moderar, los problemas crecientes que se manifiestan en la desigualdad educativa. 


\section{Más allá del modelo teórico: la evidencia empírica}

La caracterización de una pedagogía y, por derivación, de un enfoque didáctico descansa en cómo se articulan, tomando categorías de Bernstein, distintos grados de clasificación y enmarcamiento (Bernstein, 1988; 1993). Es necesario advertir que Bernstein aplica estas categorías, que expresan las relaciones de poder y los principios de control, a la constitución de los códigos, principios reguladores de la relación entre contextos y dentro de ellos (Bernstein, 1993:26 y siguientes). Aquí las retomamos para aplicarlas, según el propósito específico de este trabajo, a los formatos de enseñanza que funcionan como formas sistemáticas de ordenar la práctica educativa. Se puede decir que, como expresión de una «pedagogía», un enfoque didáctico combina una estructura de conocimiento (la clasificación del área, dominio, tema, práctica o experiencia de la que se trate) con una estructura de tarea y una regulación de la comunicación (el enmarcamiento). Incluye también, una especificación de las realizaciones aceptables (la evaluación, un aspecto que, según Bernstein, es función de cada relación particular entre clasificación y enmarcamiento). ${ }^{2}$ De esta manera, se pueden presentar diferentes combinaciones entre clasificación y enmarcamiento, dando lugar, como casos polares, a configuraciones de clasificación y enmarcamiento alto (pedagogía tradicional, visible) o de clasificación y enmarcamiento bajos (pedagogía progresista, invisible).

En momentos del avance de las llamadas pedagogías activas o de «descubrimiento» a partir de la década de 1960, que tenían como una de sus referencias el Informe Plowden y su nuevo fundamento en la teoría psicogenética de Piaget, la hipótesis de Bernstein sugería un movimiento abiertamente contrario a la corriente principal. Con el tiempo un amplio conjunto de investigaciones proveyó, mediante diferentes metodologías y escalas, y aplicadas a distintos campos de conocimiento, suficiente evidencia como para acreditar que, al menos, había un camino que ameritaba ser explorado. Si bien algunas se apoyaban directamente en la distinción entre los tipos de pedagogía que proponía Bernstein, muchas otras utilizaron otros marcos conceptuales. Pero eran coincidentes en su análisis de la influencia de los grados de estructuración de las propuestas didácticas con relación a los logros de aprendizaje.

Así, en las últimas décadas distintas investigaciones se han volcado al análisis de los efectos diferenciales que consiguen distintos modelos o enfoques didácticos con respecto a los resultados de aprendizajes. Entre estas investigaciones se pueden encontrar estudios experimentales y cuasi-experimentales de muestras pequeñas (Chase \& Klahr, 2017; Klahrl \& Nigam, 2004; Kroesbergen \& Van Luit, 2002; Kroesbergen, Van Luit \& Maas, 2004; Lorch, et al., 2010), trabajos sobre muestras muy numerosas de miles de estudiantes (Andersen \& Andersen 2017; Carabaña 2017), así como revisiones sistemáticas y meta-análisis de trabajos previamente publicados sobre el tema (Alfieri, et al., 2011; Bissonnette, 2008; Kroesbergen \& Van Luit, 2003; Hattie, 2009; Morgan, Farkas \& Maczuga, 2015). Algunas de estas investigaciones buscaron específicamente correlacionar la incidencia del enfoque didáctico con la variable de clase social o nivel socioeconómico (Andersen \& Andersen 2017; Carabaña 2017; Lubienski, 2000; 2004; Hoadley, 2005; 2007; 2008). Todos estos trabajos han partido de la comparación entre enfoques de enseñanza estructurada, directa, explícita -cuyas 
características se acercan a ciertos rasgos atribuidos a la pedagogía visible- y enfoques menos estructurados, de «guía mínima» o constructivistas -con rasgos más cercanos al modelo de pedagogía invisible- En todos los casos se encontró que los primeros son los que consiguen mejores resultados de aprendizaje en los y las estudiantes.

En la medida en que este cuerpo de trabajos desafía una de las corrientes principales de la pedagogía contemporánea, es ciertamente necesario poner en discusión sus marcos teóricos y metodológicos y los parámetros que toman en consideración para apreciar lo que se define como «mejores resultados de aprendizaje». Pero también es necesario aclarar que esta necesidad de revisión vale, del mismo modo, para las investigaciones que respalden cualquier otra orientación. Nuestro propósito consiste en sostener que existe una base suficiente para poner en cuestión la confianza indubitable en los enfoques didácticos basados en algunas versiones del constructivismo pedagógico que, con independencia de lo que acontezca realmente en las prácticas escolares, se convirtieron en los últimos treinta años en un paradigma de enorme influencia en América Latina (Braslavsky, 1999; Carnoy, 2010, Feldman \& Diuk, en prensa). Creemos que esta evidencia también avala la necesidad de explorar activamente distintas alternativas e iniciar trabajos que ofrezcan un panorama más completo.

En relación con esto último, nos parece importante remarcar que la modelización de Bernstein presentaba dos tipos polares y que esto puede conducir, fácilmente, a confrontaciones binarias entre «lo viejo»y «lo nuevo», lo «tradicional» y lo «progresivo». Y toda discusión entre modelos muy sesgados da pocas respuestas acerca de las intervenciones tendientes a la mejora de las prácticas y de sus resultados. Lo cierto es que la idea de Bernstein era que ciertas flexibilizaciones en el marco de la pedagogía visible podían actuar de un modo que favoreciera el avance escolar de los sectores populares o que, al menos, contribuyera a disminuir los rendimientos diferenciales. Un importante grupo de investigaciones se realizó en esta línea (Barret, 2017; Bohlmann, Gellert \& Straehler-Pohl, 2016; Collet-Sabé \& Martori, 2018; Ferreira, 2013; Giménez, Navas \& Graizer, 2015; Hoadley, 2007, 2008; Morais \& Neves, 2009; 2017; Piccoli, 2009; Pires, Morais \& Neves, 2004; Reeves, 2005; Sawyer, 2013). Estas investigaciones sugieren que, más que modelos polares, los formatos más favorables a los alumnos de sectores populares corresponden a formas flexibles que combinan variaciones en la fuerza de la clasificación y el enmarcamiento según sea la dimensión considerada. De ese modo, esos formatos muestran alta clasificación interna y alto enmarcamiento de la estructura de tarea, pero bajo enmarcamiento con relación al sistema de comunicación, mayor control por parte de los estudiantes con relación al ritmo y la secuencia y mayor flujo entre el conocimiento y la experiencia escolar y el conocimiento y la experiencia comunitaria. Este tipo de configuración propone una estructura básica que puede expresarse en distintas propuestas didácticas compatibles con los rasgos principales de lo que hoy se describe en la literatura internacional como «enseñanza explícita». En otro trabajo hemos argumentado que la enseñanza explícita constituye un enfoque diferenciado tanto de la enseñanza tradicional, como de los modelos progresivos o «centrados en el estudiante» (Gori, Diuk $\&$ Feldman, en revisión editorial) y que amerita ser tratado como un enfoque alternativo frente a los problemas actuales. 
En resumen, entendemos que, sin ser conclusiva, hay suficiente evidencia como para no atar las alternativas didácticas a un único conjunto de principios y creencias pedagógicas. También esta evidencia permitiría valorar de un modo amplio las maneras de colaborar más eficazmente con el avance y el éxito escolar de todos y especialmente de aquellos que hoy enfrentan más dificultades, pese al amplio abanico de políticas inclusivas desplegado en el siglo XXI. Esta tarea se revela más urgente a la luz del crecimiento de las brechas educativas producto de la situación de deterioro de las condiciones sociales, ligada con los años de proyectos neoliberales y con el actual impacto global de la situación sanitaria.

\section{De la idea de «compensación de déficit» a la necesidad de revisar el enfoque didáctico}

En la teoría de los códigos de Bernstein se describe la relación pedagógica como la realización de relaciones sociales. Estas relaciones se sintetizan en códigos que se corporizan en la estructura de clasificaciones y en los modos de comunicación entre categorías y dentro de ellas, propias de toda interacción social como la actividad escolar. De este modo, la comunicación pedagógica puede ser entendida como un medio privilegiado para la constitución/reconstrucción de relaciones sociales subordinantes y subordinadas por medio de los códigos que organizan esas relaciones de orden y jerarquía. Lo que se coloca en el centro de la escena son las relaciones de poder y sus consecuencias sobre la posibilidad de los alumnos provenientes de sectores populares de jugar en un escenario dominado por reglas que los colocan en un lugar subordinado.

Un problema siempre presente con respecto a las relaciones de poder es, en términos de Bernstein (1990), el de la articulación entre esas relaciones a nivel macro y su reproducción a nivel micro. En nuestra opinión, muchas teorías que han mantenido un enfoque crítico sobre la vinculación entre la condición social de los estudiantes y la escolaridad ofrecen un importante análisis de las «relaciones con» el sistema de reglas y principios privilegiados por la escuela y cómo los sujetos son posicionados con relación a ellas según su clase social, género o etnia. Pero es necesario explicar las «relaciones dentro» que se constituyen mediante los principios comunicativos que establecen relaciones privilegiantes en la práctica escolar. Así, identificar las distorsiones que ocurren en la comunicación pedagógica debido a influencias externas a la educación es un gran aporte, pero sería necesario, también, abordar la cuestión de la estructura interna de los principios comunicativos, el «código» según Bernstein (Bernstein, 1993; Moore, 2013). Un enfoque habitual ha sido mantener la mirada sobre las relaciones sociales como contexto de la actividad escolar, explicativo de los obstáculos que enfrentan los y las estudiantes de los sectores populares en la escuela. De allí, que los problemas escolares de los sectores populares suelen ser vistos como un problema de carencia por lo que sería necesario «compensar» ciertos «déficit» de entrada a la escuela - expresados, por ejemplo, en el «capital cultural» de origen-. Por el contrario, la hipótesis de Bernstein sugería que es en el propio planteo pedagógico donde debería buscarse un motivo de las profundas dificultades escolares que experimentan las niñas, niños y jóvenes provenientes de sectores populares y de los notorios rendimientos diferenciales en cuanto al aprendizaje si se comparan los estudiantes provenientes 
de distintos sectores. Dicho de otro modo, es necesario buscar la vinculación activa entre las condiciones sociales y los códigos de comunicación establecidos por las pedagogías en uso expresadas en distintas alternativas didácticas. Desde nuestro punto de vista, un análisis de esas características puede ayudar a diluir lo que, a nuestro juicio, es el equívoco de la compensación que parte de considerar neutral el método para concentrarse en resolver un problema «externo» y previo a la escuela.

\section{Conclusión}

En este trabajo retomamos algunos conceptos teóricos y un conjunto de investigaciones de los últimos veinte años que pueden tener valor desde el punto de vista de promover una política de enseñanza. Creemos que ese conjunto proporciona herramientas para interpretar las correlaciones entre enfoques didácticos y resultados en términos de qué es lo que facilita o dificulta la relación de los estudiantes con el código escolar. También permiten entender esas dificultades en términos didácticos, o sea internos a las propuestas escolares, y no solo de contexto, o sea externos. Esto es así, en la medida en que se comprueba el carácter para nada neutral del enfoque didáctico que se elija con relación a la posibilidad de aprendizaje y avance escolar en función de la condición social de los estudiantes. De allí que creamos que el problema educativo principal, en cuanto al rendimiento de los estudiantes provenientes de los sectores populares, no reside en un quantum de experiencias que deberían ser repuestas de manera compensadora. Porque, aunque es posible distribuir de mejor manera las condiciones materiales para la tarea y, más aún, es necesario hacerlo, el problema, según se planteó, reside en el dominio de principios de comunicación, de interacción y de orientación al significado diferentes. Por lo tanto, se requiere un principio de orden y regulación con el que puedan interactuar exitosamente los estudiantes de los sectores populares en la progresiva adquisición de competencia.

Por último, la evidencia disponible muestra que es momento de abrir un debate amplio sobre los enfoques más prestigiosos y los principios en los que se sustentan. Las preocupaciones actuales acerca del aprovechamiento escolar de amplios sectores de estudiantes parecen aconsejar la conveniencia de poner a prueba distintas propuestas didácticas para verificar su efectivo papel y su eficacia en términos de aprendizajes en general y, en particular, sobre el diferencial de aprendizajes ligado con la condición social. Pensamos que es necesario llevar adelante programas comparativos que estudien las bondades y debilidades de las estrategias y métodos juzgados como deseables y avalen sus conclusiones en datos, más que en apreciaciones ligadas con valores polares como innovación, tradicionalismo, tecnicismo, creatividad, autoritarismo, libertad, memorístico, significativo, constructivista, pasividad, etc. Sería necesario revisar la confianza en ciertas propuestas de enseñanza basada, principalmente, en sus fundamentos teóricos fuertes y en los componentes valorativos que estos conllevan. Tal confianza no está exenta del riesgo de que se insista en asegurar el valor de ciertas prácticas con independencia de contar con evidencias suficientes sobre el cumplimiento real de los altos propósitos enunciados. Hace falta, en conclusión, relacionar activamente didáctica con condición social y valorar las propuestas en 
función de su eficacia para cumplir con los principios de derecho al aprendizaje y de acceso equitativo a la educación.

\section{Referencias}

Alfieri, L., Brooks, P. J., Aldrich, N. J., \& Tenenbaum, H. R. (2011). Does discoverybased instruction enhance learning? Journal of Educational Psychology, 103(1), 118. https://doi.org/10.1037/a0021017

Andersen, I. G., \& Andersen, S. C. (2017). Student-Centered Instruction and Academic Achievement: Linking Mechanisms of Educational Inequality to Schools' Instructional Strategy. British Journal of Sociology of Education, 38 (4), 533-550. https://doi.org/10.1080/01425692.2015.1093409

Barrett, B. D. (2017). Bernstein in the urban classroom: a case study. British Journal of Sociology of Education, 38 (8), 1258-1272. https://doi.org/10.1080/01425692.2 016.1269632 .

Bernstein, B. (1988). Clase y pedagogía: visible e invisible. En Bernstein, B. Clases, códigos y control (pp.109-144). Madrid: Akal.

Bernstein, B. (1990). Poder, educación y conciencia. Sociología de la transmisión cultural. Barcelona: El Roure Editorial, S. A.

Bernstein, B. (1993). La estructura del discurso pedagógico. Madrid: Morata.

Bissonnette, S. (2008). Réforme éducative et stratégies d'enseignemment: synthese de recherches sur l'efficacité de l'enseignemment et des ecoles. (Tesis Doctoral) Departemment d'études sur l'énseignement et l'apprentissage. Faculté des Sciences de L'Education. Université Laval. Quebec, Canadá. https://corpus.ulav al.ca/jspui/handle/20.500.11794/19958

Bohlmann, N.; Gellert, U. \& Straehler-Pohl, H. (2016). Investigando las desigualdades sociales en el aula de matemáticas: logros y expectativas. Didacticae I, 26-44. https://doi.org/10.1344/did.2017.1.26-44

Braslavsky, C. (1999). The Secondary Education Curiculum in Latin America: New Tendencies and Changes. Final Report of the Seminar Organized by the International Bureau of Education. International Institute for Educational Planing. Buenos Aires, Argentina, September 2-3.

Carabaña J. (2017). Clase social de origen y didáctica de las matemáticas. Revista Tempora, 19, 17-50.

Carnoy, M. (2010) La ventaja Académica de Cuba. Fondo de Cultura Económica

Cavanaugh, C.; Ae-Hwa, K.; Wanzek, C. \& Vaughn, S. (2004). Kindergarten Reading Interventions for At-Risk Students: Twenty Years of Research. Learning Disabilities: A Contemporary Journal, 2 (1), pp. 9-21. https://digilander.libero.i t/adriauno/metareadatrisk04.pdf

Chase, C.C., \& Klahr, D. (2017). Invention Versus Direct Instruction: For Some Content, It's a Tie.J Sci Educ Technol, 26, pp. 582-596. https://doi.org/10.100 7/s10956-017-9700-6

Collet-Sabé, J. \& Martori, J. C. (2018). Bridging boundaries with Bernstein: approach, procedure and results of a school support project in Catalonia, British Journal of Sociology of Education, 39 (8), 1126-1142. https://doi.org/10.1080/01425692.2 018.1478718

Feldman, D. \& Diuk, B. (en prensa). Políticas de enseñanza y desigualdad: entre los formatos pedagógicos y las finalidades educativas. Revista Educativa. 
Ferreira, E. (2013). A practica pedagogica como o diferencial para um desempenho escolar efetivo - um estudo abordando as séries finais do ensino fundamental em escolas do periferia urbana de Porto Alegre. (Tesis de Doctorado). Facultade do Educaçao, Universidade Federeal do Rio Grande do Sul. https://www.lume.ufrgs.br/handl e/10183/70588

Giménez, E.; Navas, A. \& Graizer, O. (2015). ¿Puede la educación transformar el orden social? Descripción de una modalidad pedagógica que permite el acceso a posiciones sociales diferenciales. Profesorado. Revista de Currículum y Formación de Profesorado, 19 (3), 94-109. https://recyt.fecyt.es/index.php/profesorado/ article/view/43631

Gori, A., Diuk, B. \& Feldman, D. (manuscrito bajo revisión editorial). La enseñanza explícita en la discusión didáctica actual.

Graizer, O. L. \& Navas Saurin, A. (2011). El uso de la teoría de Basil Bernstein como metodología de investigación en Didáctica y Organización Escolar. Revista de Educación, 356, 133-158. https://recyt.fecyt.es/index.php/Redu/article/view/10 913

Hattie (2009). Visible Learning. A synthesis of over 800 meta-analyses relating to achievement. London: Routledge.

Hoadley, U. (2005). Social class, pedagogy, and the specialization of voice in four South African primary schools. (PhD Thesis). University of Cape Town, South Africa. h ttps://open.uct.ac.za/handle/11427/8659

Hoadley, U. (2007). The reproduction of social class inequalities through mathematics pedagogies in South African primary schools. Journal of Curriculum Studies, 39 (6), 679-706. https://doi.org/10.1080/00220270701261169

Hoadley, U. (2008). Social class and pedagogy: a model for the investigation of pedagogic variation. British Journal of Sociology of Education, 29 (1), 63-78. http s://doi.org/10.1080/01425690701742861

Klahrl, D. \& Nigam, M. (2004). The equivalence of learning paths in early science instruction: Effects of direct instruction and discovery learning. Psychological Science, 15 (10), 661-667. https://doi.org/10.1111/j.0956-7976.2004.00737.x

Kroesbergen, E. H. \& Van Luit, J. E. H. (2002) Teaching multiplication to low math performers: Guided versus structured instruction. Instructional Science 30, 361378, https://doi.org/10.1023/A:1019880913714

Kroesbergen, E. H. \& Van Luit, J. E. H. (2003). Mathematics Interventions for Children with Special Educational Needs. A Meta-Analysis. Remedial and Special education, 24 (2), 97-114. https://doi.org/10.1177/07419325030240020501

Kroesbergen, E. H., Van Luit, J. E., \& Maas, J. E. (2004). Effectiveness of explicit and constructivist mathematics instruction for low-achieving students in the Netherlands. The Elementary School Journal, 104 (3), 233-251. https:// doi.org/10.1086/499751

Lubienski, S. T. (2000). Problem Solving as a Means toward Mathematics for All: An Exploratory Look through a Class Lens. Journal for Research in Mathematics Education, 31 (4), 454-482. https://doi.org/10.2307/749653

Lubienski, S. T. (2004). Decoding mathematics instruction. A critical examination of an invisible pedagogy. En: Davis, B.; Muller, J. \& Morais, A. M. (2004) Reading Bernstein Researching Bernstein. (108-122) Routledge Falmer. 2004.

Lorch Jr, R. F., Lorch, E. P., Calderhead, W. J., Dunlap, E. E., Hodell, E. C., \& Freer, B. D. (2010). Learning the control of variables strategy in higher and lower achieving classrooms: Contributions of explicit instruction and experimentation. Journal of Educational Psychology, 102(1), 90-101. https://doi.org/10.1037/a0017972 
Moore, R. (2013). Basil Bernstein. The thinker and the field. London: Routledge.

Morais, A. M. \& Neves, I. P. (2009). Textos e contextos educativos que promovem aprendizagem - optimização de um modelo de prática pedagógica. Revista Portuguesa de Educação, 22 (1), 5-28.

Morais, A. M. \& Neves, I. P. (2017). The quest for high level knowledge in schools. Revisiting the concepts of classification and framing. British Journal of Sociology of Education, 39 (3), 261-282. https://doi.org/10.1080/01425692.2017.1335590

Morgan, P. L.; Farkas, G. \& Maczuga, S. (2015). Which Instructional Practices Most Help First-Grade Students with and without Mathematics Difficulties? Educational Evaluation and Policy Analysis, 37 (2), 184 - 205. https://doi.org/1 $0.3102 / 0162373714536608$

Piccoli, L. (2009). Práctica Pedagógica nos Processosde Alfabatizacao e de Letramento: análises a partir dos campos da sociologia e da linguagem. (Tesis Doctoral). Universidad Federal do Rio Grande do Sul, Brasil. https://www.lume.ufrgs.br/h andle/10183/18400

Pires, D.; Morais, A. M. \& Neves I. P. (2004). Desenvolvimento cientifico nos primeiros anos de escolaridade. Revista de Educacao em Educacao, 12(2), 129-156.

Reeves, C. A. (2005). The effect of «opportunity to learn» and classroom pedagogy on mathematics achievement in schools serving low socio-economic status communities in the Cape Peninsula. (Tesis de Doctorado). School of Education Faculty of Humanities, University of Cape Town. https://open.uct.ac.za/handle/11427/1 0895

Rupley, W. H.; Blair, T. R. \& Nichols, W. D. (2009). Effective Reading Instruction for Struggling Readers: The Role of Direct/Explicit Teaching, Reading \& Writing Quarterly: Overcoming Learning Difficulties, 25 (2-3), 125-138. https://doi.org/ $10.1080 / 10573560802683523$

Ryder, J. F., Tunmer, W. E., \& Greaney, K. T. (2008). Explicit instruction in phonemic awareness and phonemically based decoding skills as an intervention strategy for struggling readers in whole language classrooms. Reading and Writting: An Interdisciplinary Journal, 21 (4), 349-369. https://doi.org/10.1007/s11145-007 $-9080-\mathrm{z}$

Sawyer, A. (2013). Student identities in mathematical community of practice: radical visible pedagogy and the teacher collective. (Tesis de Doctorado). Queensland University of Technology. https://eprints.qut.edu.au/63818/

Xue, Y. \& Meisels, S. J. (2004). Early Literacy Instruction and Learning in Kindergarten: Evidence from the Early Childhood Longitudinal Study-- Kindergarten Class of 1998-1999. American Educational Research Journal, 41 (1), 191-229. https://do i.org/10.3102/00028312041001191

\section{Notas}

1 De hecho, muchas familias que eligen modelos de pedagogía invisible para la primera escolaridad prefieran modelos de pedagogía visible para la escuela secundaria cuando los desempeños específicos pasan a tener más importancia por el acceso a los estudios superiores)

2 El propio Bernstein establece una vinculación bastante directa entre los modelos generales de «pedagogías visibles e invisibles» y distintas teorías instruccionales que, puede decirse, se concretan en enfoques y estrategias didácticas. Por ejemplo, en Bernstein (1993:82). 


\section{Notas de autor}

Sobre el autor

Lic. en Ciencias de la Educación - Universidad de Buenos Aires. Departamento de Ciencias de la Educación, IICE. Facultad de Filosofía y Letras.

Sobre el autor

Lic. en Ciencias de la Educación - Universidad de Buenos Aires. Becario doctoral CONICET.

Sobre la autora

Lic. en Ciencias de la Educación - UBA/ Doctora en Psicología - UNLP. LICH/ UNSAM-CONICET. 